\title{
Three-Dimensional Display Utilizing a Diffractive Optical Element and an Active Matrix Liquid Crystal Display
}

Gregory P. Nordin

nordin@byu.edu

M. W. Jones

S. T. Kowel

J. H. Kulick

R. G. Lindquist

Follow this and additional works at: https://scholarsarchive.byu.edu/facpub

Part of the Electrical and Computer Engineering Commons

\section{Original Publication Citation}

G. P. Nordin, M. W. Jones, J. H. Kulick, R. G. Lindquist, and S. T. Kowel, "A 3-D Display Utilizing a Diffractive Optical Element and an Active Matrix Liquid Crystal Display," Opt. Eng. 35(12), pp. 344-3412 (1996)

\section{BYU ScholarsArchive Citation}

Nordin, Gregory P.; Jones, M. W.; Kowel, S. T.; Kulick, J. H.; and Lindquist, R. G., "Three-Dimensional Display Utilizing a Diffractive Optical Element and an Active Matrix Liquid Crystal Display" (1996). Faculty Publications. 1146.

https://scholarsarchive.byu.edu/facpub/1146

This Peer-Reviewed Article is brought to you for free and open access by BYU ScholarsArchive. It has been accepted for inclusion in Faculty Publications by an authorized administrator of BYU ScholarsArchive. For more information, please contact ellen_amatangelo@byu.edu. 


\section{Three-dimensional display utilizing a diffractive optical element and an active matrix liquid crystal display}

\author{
Gregory P. Nordin \\ Michael W. Jones \\ Jeffrey H. Kulick, MEMBER SPIE \\ Robert G. Lindquist \\ Stephen T. Kowel, MEMBER SPIE \\ The University of Alabama in Huntsville \\ Department of Electrical and \\ Computer Engineering \\ Huntsville, Alabama 35899
}

\begin{abstract}
We describe the design, construction, and performance of the first real-time autostereoscopic three-dimensional (3-D) display based on the partial pixel 3-D display architecture. The primary optical components of the 3-D display are an active-matrix liquid crystal display and a diffractive optical element (DOE). The display operates at video frame rates and is driven with a conventional VGA signal. Three-dimensional animations with horizontal motion parallax are readily viewable as sets of stereo images. Formation of the virtual viewing slits by diffraction from the partial pixel apertures is experimentally verified. The measured contrast and perceived brightness of the display are excellent, but there are minor flaws in image quality due to secondary images. The source of these images and how they may be eliminated is discussed. The effects of manufacturing-related systematic errors in the DOE are also analyzed. (C) 1996 Society of Photo-Optical Instrumentation Engineers.
\end{abstract}

Subject terms: electronic holography; liquid crystal displays; diffractive optics; diffractive optical elements; three-dimensional displays.

Paper 25066 received Feb. 15, 1996; revised manuscript received June 7, 1996; accepted for publication June 20,1996. This paper was originally submitted for the special section on Electronic Holography in June 1996, but was not available in time.

\section{Introduction}

Binocular disparity and motion parallax are critical physiological cues for depth perception in the human visual system. ${ }^{1,2}$ It is difficult, however, to provide both of these cues simultaneously in a real-time autostereoscopic (i.e., no head gear required) display device. Over the past few years, a number of efforts to implement real-time holographic display systems, which inherently provide these depth cues, have been reported. ${ }^{3-8}$ Several parallel efforts toward holographic-like displays based on the use of various diffractive elements are also being pursued. ${ }^{9-13}$ In this paper we discuss such an approach in which we have achieved horizontal-only motion parallax and binocular disparity in a real-time autostereoscopic display based on our partial pixel 3-D display architecture. ${ }^{10}$ This small demonstration display is functionally equivalent to a real-time holographic stereogram. It uses a conventional liquid crystal display (LCD) in conjunction with a diffractive optical element (DOE).

A key innovation of our approach is that the high space bandwidth product (SBWP) features required to diffract light from the readout beam into the viewing region are fixed in the DOE (and thus must only be computed and generated once), while the lower SBWP information that constitutes a 3-D scene (in the form of a set of 2-D stereo image pairs) is displayed at video frame rates on the LCD. Other features of the partial pixel architecture include the use of incoherent illumination and straightforward extension to full-color operation. ${ }^{10,14,15}$
The small, limited-resolution monochrome display described in this paper is our first real-time implementation of the partial pixel 3-D display architecture. ${ }^{12}$ As such, it demonstrates the visual feasibility of creating real-time autostereoscopic 3-D displays that exhibit horizontal motion parallax as well as binocular disparity. Ultimately, the creation of partial pixel architecture 3-D displays with the size and resolution of typical computer monitors requires the development of LCDs that have very large numbers of pixels such as are being developed by Xerox. ${ }^{16}$ The demonstration display discussed in this paper thus represents a first step toward potentially practical 3-D displays that function like real-time holographic stereograms.

We begin in Sec. 2 with a brief review of the key concepts of the partial pixel architecture (a detailed discussion is provided in Ref. 10). The design and construction of the demonstration display are discussed next in Sec. 3. This includes an analysis of the effects of DOE fabrication errors. In Sec. 4 the performance of the display is evaluated. We then discuss future research directions.

\section{Partial Pixel Architecture}

The basic geometry of the partial pixel architecture is illustrated in Fig. 1. It consists of a pixelated display and a viewing region located at a distance $D_{v}$ from the display. As shown in the figure, the viewing region is composed of a series of virtual viewing slits, each of which has a width of approximately one pupil diameter. A unique 2-D image is visible on the display from each of these virtual viewing slits. When an appropriate pair of 2-D images (a stereoimage pair) is seen simultaneously by the left and right 


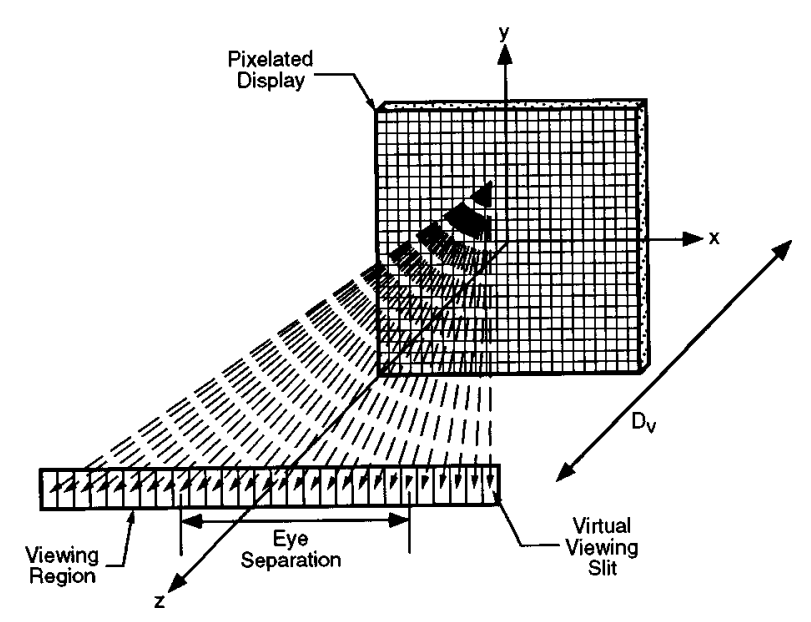

Fig. 1 Schematic diagram of the geometry of the partial pixel 3-D display architecture (after Ref. 10). As discussed in the text, light from each pixel illuminates each of the virtual viewing slits (illustrated for a single pixel).

eyes, the scene appears three-dimensional. Horizontal motion parallax is provided by simultaneously displaying a suitable set of stereo-image pairs, with each image visible from a single virtual viewing slit.

In order to simultaneously display multiple 2-D images, each pixel in the display must have an independent appearance when viewed from each of the virtual viewing slits. This is accomplished by dividing each pixel into spatially distinct regions that we refer to as partial pixels. ${ }^{10}$ There is a one-to-one correspondence between partial pixels and virtual viewing slits. Each partial pixel in a given pixel is responsible for the pixel's overall appearance when viewed from its corresponding virtual viewing slit. This is possible because the size of each pixel is designed to be at or below the resolution limit of the human eye when the display is looked at from the viewing region.

A unique diffraction grating is used in each partial pixel to direct light to the appropriate virtual viewing slit. Methods of modulating the light that passes through individual partial pixels include (1) use of a separate conventional liquid crystal display ${ }^{12}$ and (2) varying the efficiency of the diffraction gratings. ${ }^{11,17}$ The display described herein uses the first method. As discussed in Ref. 10, diffraction from the partial pixel apertures is used to define the physical size of the virtual viewing slits. Hence the required partial pixel aperture size for a given display is derived from the viewing geometry and the desired virtual viewing slit size.

\section{Device Design and Construction}

In this section we discuss the design and construction of our small demonstration 3-D display. We begin with a brief overview of the display and viewing region geometries followed by a description of the liquid crystal display used for light modulation. Next we describe the diffractive optical element. This includes an examination of the effects of potential systematic errors in the grating pitch and angular orientation introduced in the manufacturing process. Finally, we conclude this section by outlining how 3-D animations are prepared and viewed on the display.

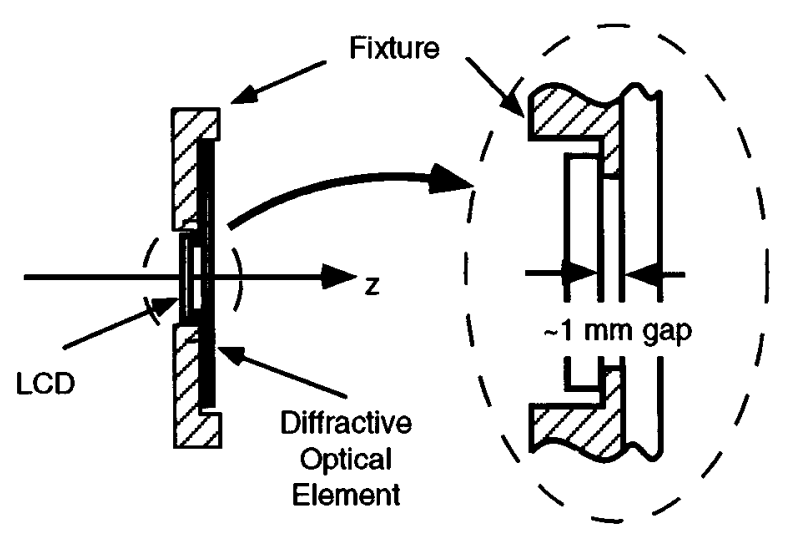

Fig. 2 Schematic diagram of the alignment fixture for the liquid crystal display and the diffractive optical element (after Ref. 12).

\subsection{Device Overview}

The primary components of our prototype display are a diffractive optical element (DOE) to direct light from each partial pixel to its corresponding virtual viewing slit and a separate conventional liquid crystal display (LCD) to modulate the light. ${ }^{12}$ Each pixel in the LCD is used to modulate the intensity of the light passing through a single diffraction grating in the DOE. This requires precise spatial registration of the LCD pixel apertures relative to the gratings. As illustrated in Fig. 2, both the DOE and the LCD are mounted in a fixture that allows them to be aligned relative to each other to within a few microns. The crossed polarizers, which are traditionally placed on both surfaces of the LCD, are positioned on either side of the LCD/DOE assembly to allow the DOE to be placed in close proximity to the LCD.

To avoid confusion with terminology in the ensuing sections, the following definitions will be used. A pixel in the LCD will be referred to as an LCD pixel. Each partial pixel consists of a single LCD pixel and a single diffraction grating in the DOE. A display pixel is an array of partial pixels with the number of partial pixels equal to the number of virtual viewing slits that are used in the viewing region.

\subsection{Display and Viewing Region Geometries}

The active area of the display is approximately $2 \times 3 \mathrm{~cm}$. As illustrated in Figs. 3 and 4, the display is designed to be illuminated from the back at an angle of $10 \mathrm{deg}$ with a collimated incoherent quasi-monochromatic beam centered at a wavelength of $630 \mathrm{~nm}$. The viewing region is $30 \mathrm{~cm}$ from the display and is divided into 19 virtual viewing slits, of which the three central slits are unused. Each virtual viewing slit is approximately $6 \mathrm{~mm}$ wide and $10 \mathrm{~mm}$ tall. The eight left-most virtual viewing slits are intended to be seen by an observer's left eye, and similarly the eight rightmost virtual viewing slits are intended for the right eye.

The display is composed of a $135 \times 90$ array of display pixels, each of which is $220 \times 220 \mu \mathrm{m}^{2}$. As illustrated in Fig. 5, each partial pixel aperture is approximately 40 $\mu \mathrm{m} \times 25 \mu \mathrm{m}$ and is defined by the overlap of the grating aperture and the LCD pixel aperture. Each display pixel is 


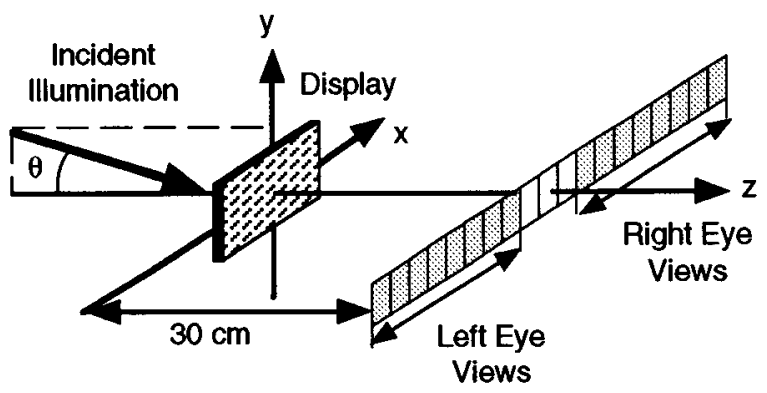

Fig. 3 Schematic diagram of the display readout geometry. The display consists of the LCD/DOE assembly placed between crossed polarizers.

composed of a $4 \times 4$ array of such partial pixels as shown in Fig. 5(a). The number in each partial pixel in this figure is the index of the virtual viewing slit to which it directs light. Referring to Fig. 3, the index of the far left-hand slit is -9 while that of the far right-hand slit is +9 .

\subsection{Liquid Crystal Display}

The LCD is a miniature $(35.2 \times 26.4 \mathrm{~mm})$ VGA-compatible $(640 \times 480$ pixels) monochrome active matrix liquid crystal display (AMLCD) with gray scale capability manufactured by Kopin Corporation. It was chosen primarily for two reasons. First, the drive electronics permit individual LCD pixels to be independently addressed with a standard VGA input signal. Second, to adequately fill the virtual viewing slits with light using diffraction from the partial pixel apertures, the aperture width must be on the order of $40 \mu \mathrm{m}$ for our display geometry. The Kopin LCD pixels, which have a clear aperture of approximately $40 \times 40 \mu \mathrm{m}^{2}$ on a $55-\mu \mathrm{m}$ pitch, are ideal for this purpose.

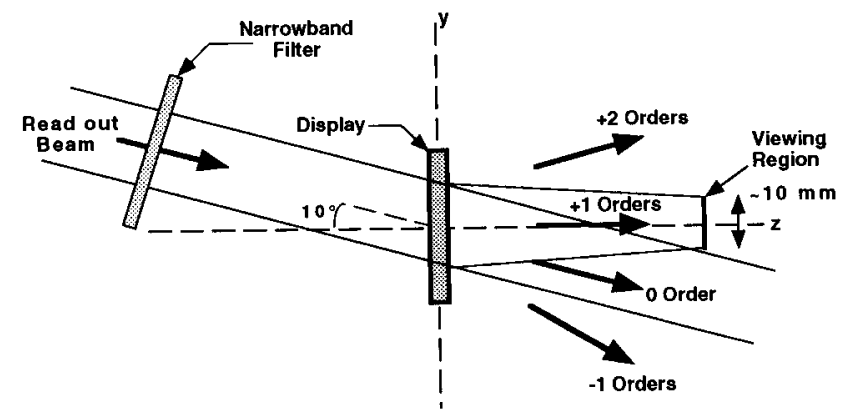

Fig. 4 Side view of the display readout geometry illustrating the directions of the undiffracted readout beam and the grating diffraction orders.

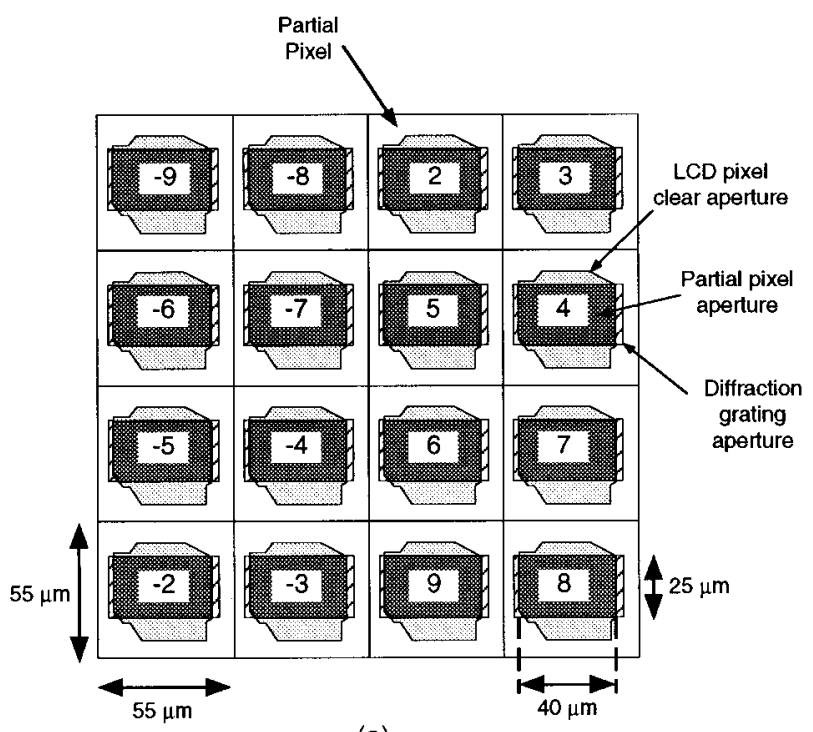

(a)

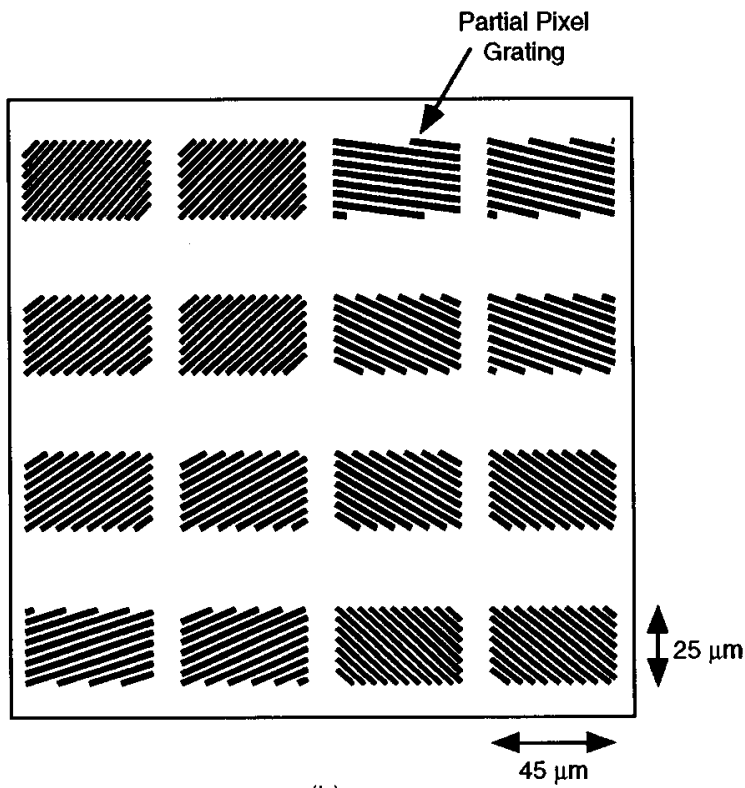

(b)

Fig. 5 (a) Schematic diagram of a single display pixel composed of a $4 \times 4$ array of partial pixels. The number in each partial pixel is a virtual viewing slit index as described in the text; (b) corresponding array of DOE gratings. Each grating on the DOE has a unique angular orientation and grating pitch.

\subsection{Diffractive Optical Element}

\subsubsection{Design}

The diffractive optical element is implemented as a fixed set of $50 \%$ duty cycle amplitude gratings etched in a chrome-on-quartz mask. The gratings on the DOE cover an area of approximately $2 \times 3 \mathrm{~cm}^{2}$ and thus define the active area of the display. There are 194,400 unique gratings with feature sizes ranging from 1.05 to $2.25 \mu \mathrm{m}$. The specific period and angular orientation of each grating were designed as outlined in Ref. 10 to direct the +1 diffraction order to the appropriate virtual viewing slit. As illustrated in Fig. 4, the undiffracted light and the other diffraction 


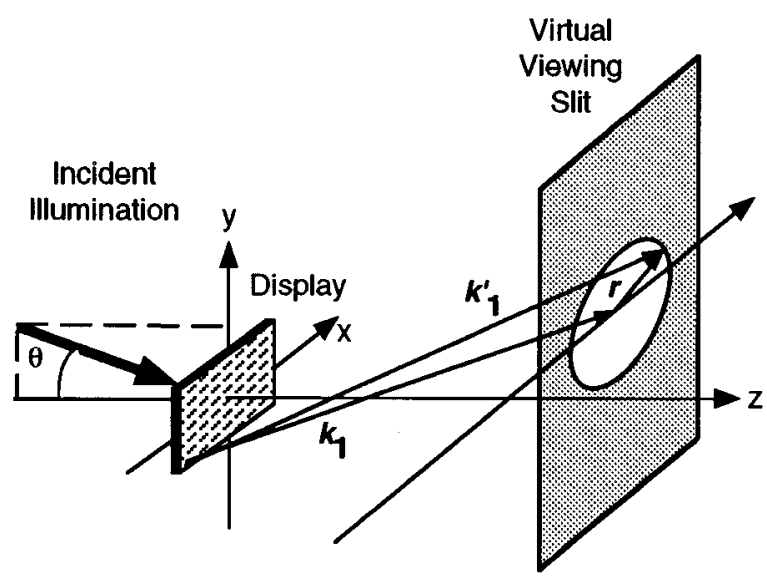

Fig. 6 Position error, $r$, in the plane of the viewing region caused by grating pitch and angular orientation errors. The ideal diffracted beam, $k_{1}$, is directed toward the center of the appropriate virtual viewing slit, while the actual diffracted beam, $k^{\prime}{ }_{1}$, is displaced by $r$.

orders do not intersect the viewing region and hence do not affect the visual quality of the display. The grating height $(25 \mu \mathrm{m})$ was chosen to yield a virtual viewing slit height of approximately $10 \mathrm{~mm}$. The horizontal dimension of the grating is $45 \mu \mathrm{m}$ and was selected to provide a 5- $\mu \mathrm{m}$ margin in the horizontal alignment of the DOE to the LCD. The gratings for a representative display pixel are shown in Fig. $5(b)$.

\subsubsection{Grating error analysis}

The DOE manufacturing process can introduce potential systematic errors in the period of the gratings. For example, the DOE is fabricated as six individual $1-\mathrm{cm}^{2}$ cells, each of which is first patterned at $10 \times$ using a vector-scan laser plotter. The six $10 \times$ reticles are then photoreduced (each to an area of approximately $1 \mathrm{~cm}^{2}$ ) onto a final chrome-onquartz mask using standard photolithographic techniques. In general, there is some residual difference in the magnification of the photoreduction step compared with the ideal magnification. This residual error is usually slightly different for each reticle. Furthermore, the presence of aberrations in the photoreduction optics causes a small variation in the magnification across the image of each reticle at the plane of the mask. This effect is most pronounced at the edges of each $1-\mathrm{cm}^{2}$ image field. The net result is a potential systematic error in the grating pitch for any given partial pixel on the fabricated mask compared with the corresponding ideal partial pixel grating. This in turn can result in a misdirected diffracted beam, which can cause crosstalk between images in the viewing region.

An additional consideration in minimizing image crosstalk in the viewing region is the required accuracy of the angular orientation of each grating, which is determined by the accuracy of the vector-scan laser plotter. In this subsection we develop an error model that includes variations in both the grating pitch and angular orientation. As illustrated in Fig. 6, our primary goal is to calculate the magnitude of the position error, $r$, of the diffracted beam as it

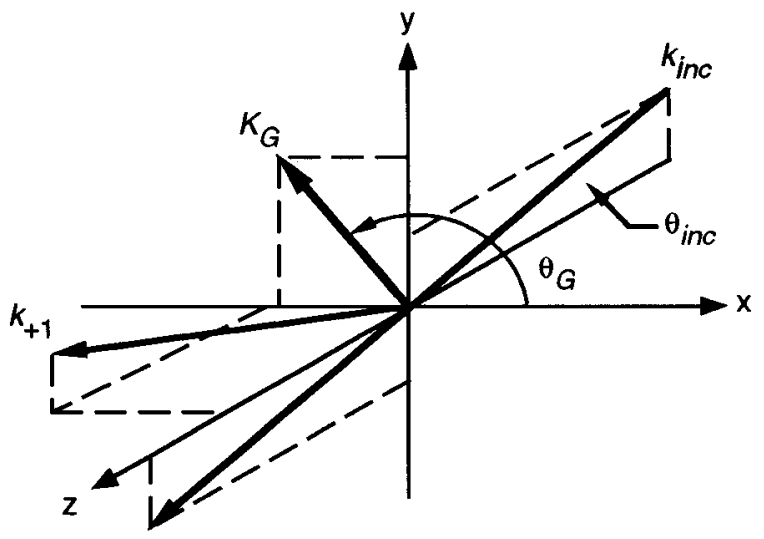

Fig. 7 Geometry of readout beam, partial pixel grating wave vector, and the +1 order diffracted beam.

intersects the plane of the viewing region. This allows us to set requirements for the grating pitch and angular orientation fabrication tolerances.

Consider the diffraction grating geometry in Fig. 7. A collimated monochromatic readout beam is incident on a grating that is confined to the $x-y$ plane. The propagation direction of the readout beam is in the $y-z$ plane at an angle $\theta_{\text {inc }}$ from the $z$ axis. The diffraction grating wave vector is

$\mathbf{K}_{\mathbf{G}}=K\left[\left(\cos \theta_{G}\right) \hat{x}+\left(\sin \theta_{G}\right) \hat{y}\right]$,

in which $K=2 \pi / \Lambda, \Lambda$ is the grating pitch, and $\theta_{G}$ is the angular orientation of the grating as shown in Fig. 7. A systematic error in the grating pitch and angular orientation yields a grating wave vector of

$\mathbf{K}_{\mathbf{G}}^{\prime}=(K+\Delta K)\left\{\left[\cos \left(\theta_{G}+\Delta \theta\right)\right] \hat{x}+\left[\sin \left(\theta_{G}+\Delta \theta\right)\right] \hat{y}\right\}$,

in which $\Delta \theta$ is the error in the angular orientation and $\Delta K$ is related to the error in the grating pitch, $\Delta \Lambda$, by

$\Delta K=-K \frac{\Delta \Lambda / \Lambda}{1+\Delta \Lambda / \Lambda}$

The components of the wave vector of the +1 diffracted order produced by the grating of Eq. (2) are

$k_{1 x}^{\prime}=(K+\Delta K) \cos \left(\theta_{G}+\Delta \theta\right)$

$k_{1 y}^{\prime}=k_{\mathrm{inc}} \sin \theta_{\mathrm{inc}}+(K+\Delta K) \sin \left(\theta_{G}+\Delta \theta\right)$

$k_{1 z}^{\prime}=k_{\text {inc }}\left(1-\alpha_{1}^{\prime 2}-\beta_{1}^{\prime 2}\right)^{1 / 2}$,

in which 
$k_{\text {inc }}=\frac{2 \pi}{\lambda}, \quad \alpha_{1}^{\prime}=\frac{k_{1 x}^{\prime}}{k_{\text {inc }}}, \quad \beta_{1}^{\prime}=\frac{k_{1 y}^{\prime}}{k_{\text {inc }}}$,

and $\lambda$ is the wavelength of the readout illumination. This +1 order beam intersects the plane of the viewing region at the point given by

$x^{\prime}=\frac{k_{1 x}^{\prime}}{k_{1 z}^{\prime}} D_{\nu}=\frac{D_{\nu}}{k_{1 z}^{\prime}}(K+\Delta K) \cos \left(\theta_{G}+\Delta \theta\right)$

$y^{\prime}=\frac{k_{1 y}^{\prime}}{k_{1 z}^{\prime}} D_{\nu}=\frac{D_{\nu}}{k_{1 z}^{\prime}}\left[k_{\text {inc }} \sin \theta_{\text {inc }}+(K+\Delta K) \sin \left(\theta_{G}+\Delta \theta\right)\right]$.

The beam diffracted by the ideal grating intersects the plane of the viewing region at the point

$x=\frac{k_{1 x}}{k_{1 z}} D_{\nu}=\frac{D_{\nu}}{k_{1 z}} K \cos \theta_{G}$

$y=\frac{k_{1 y}}{k_{1 z}} D_{\nu}=\frac{D_{\nu}}{k_{1 z}}\left[k_{\mathrm{inc}} \sin \theta_{\mathrm{inc}}+K \sin \theta_{G}\right]$.

Assuming that $\Delta K$ and $\Delta \theta$ are small compared with $K$ and $\theta_{G}$ (so that $k_{1 z}^{\prime} \approx k_{1 z}$ ), the displacement of the actual beam from the ideal beam in the plane of the viewing region can be written as

$\Delta x=x^{\prime}-x \cong \frac{D_{\nu}}{k_{1 z}}\left[(K+\Delta K) \cos \left(\theta_{G}+\Delta \theta\right)-K \cos \theta_{G}\right]$

$\Delta y=y^{\prime}-y \cong \frac{D_{\nu}}{k_{1 z}}\left[(K+\Delta K) \sin \left(\theta_{G}+\Delta \theta\right)-K \sin \theta_{G}\right]$.

The magnitude of the position error is given by $r=\left(\Delta x^{2}+\Delta y^{2}\right)^{1 / 2}$. Keeping the lowest-order terms in $\Delta \theta$ and $\Delta K$, this can be expressed as

$r \cong D_{\nu} \frac{K}{k_{1 z}}\left[(\Delta \theta)^{2}+\left(\frac{\Delta K}{K}\right)^{2}\right]^{1 / 2}$.

Replacing $\Delta K$ with Eq. (3) and again keeping lowest-order terms, the magnitude of the position error becomes

$r \cong D_{\nu} \frac{K}{k_{1 z}}\left[(\Delta \theta)^{2}+\left(\frac{\Delta \Lambda}{\Lambda}\right)^{2}\right]^{1 / 2}$.

Inspection of Eq. (10) shows that the position error is greatest for given pitch and angular orientation errors, $\Delta \Lambda$ and $\Delta \theta$, when the grating pitch is smallest. The maximum position error can therefore be determined by considering the partial pixel that contains the grating with the smallest pitch. This occurs for the partial pixel located at either the bottom right corner pixel of the display that directs light into the extreme left virtual viewing slit or the bottom left corner pixel of the display that directs light into the extreme right virtual viewing slit.

The maximum acceptable position error for the diffracted beams can be determined from the following somewhat arbitrary requirements. First, the maximum error should be less than approximately $10 \%$ of the width of the virtual viewing slits in order to limit crosstalk between slits. Second, the center of each diffracted beam should be incident on the pupil of an observer when the pupil is centered in the virtual viewing slit (i.e., the position error should be less than one-half the diameter of the pupil, which is typically $\sim 3 \mathrm{~mm}$ ). This is to ensure brightness uniformity among the partial pixels visible from each virtual viewing slit. Since the width of the virtual viewing slit for our prototype display is $6 \mathrm{~mm}$, the first guideline is the more restrictive of the two and requires that $r \leqslant 0.6 \mathrm{~mm}$.

In the mask fabrication process, the maximum pitch and angular orientation errors are, respectively, $0.01 \mu \mathrm{m}$ and $0.001 \mathrm{deg} .{ }^{18}$ The minimum grating pitch in the DOE is 2 $\mu \mathrm{m}$. The maximum position error is thus $\sim 0.5 \mathrm{~mm}$, which is within the above tolerance. Note that this is the maximum error, and that the typical error will be smaller. Hence we expect the display to exhibit well-formed slits, which is exactly what we observe, as discussed in Sec. 4.3.

\subsection{Animation Preparation}

The animated sequences displayed on the device are precomputed and stored as a standard VGA-compatible movie file. Each frame of the animation is generated by defining the 3-D scene for that frame and then calculating the 2-D projections of the scene onto the plane of the display for each virtual viewing slit. The multiple $2-\mathrm{D}$ images are then interlaced according to the virtual viewing slit index allocation indicated in Fig. 5(a) and stored on disk as a single frame. After this process is repeated for each frame, the individual frames are compiled into a single movie file. The display is then driven with a VGA signal generated on a 486 PC to display the movie file.

\section{Performance Evaluation}

In this section we describe the performance of our prototype display. We first examine the qualitative performance of the display and discuss the presence of secondary images and how they may be eliminated. Next we evaluate the contrast ratio and optical efficiency of the display. We conclude the section by examining the intensity profile of the viewing region to verify that the viewing region and the individual virtual viewing slits are formed as predicted.

\subsection{Qualitative Evaluation}

The device was illuminated as illustrated in Figs. 3 and 4 with a highly collimated beam from a mercury vapor arc lamp. A 10-nm bandpass filter centered at $630 \mathrm{~nm}$ was placed before the display as shown in Fig. 4. The display was viewed from the viewing region while a precomputed movie was displayed. The animated 2-D images were bright, clearly visible, and easily fused into a 3-D scene. Horizontal motion parallax was also distinctly discernible as the observer traversed the viewing region. However, sec- 


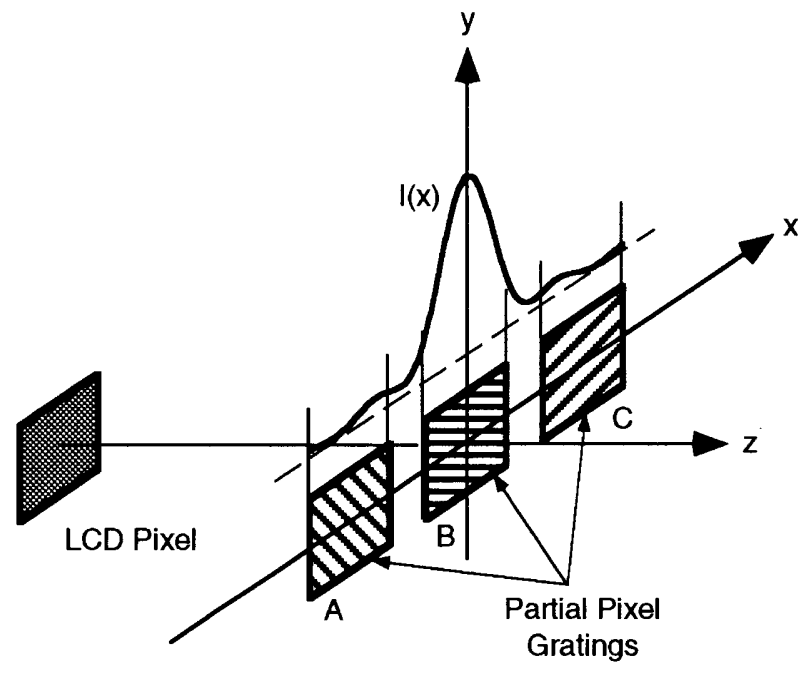

Fig. 8 Schematic diagram of a single LCD pixel and three horizontally adjacent partial pixel gratings on the DOE. The plot is the $x$ axis intensity profile at the DOE due to diffraction from the LCD pixel aperture.

ondary images were visible in most of the virtual viewing slits, which resulted in a form of interocular crosstalk. ${ }^{19}$ These were somewhat distracting to the viewer and degraded the overall visual quality of the display.

We find that the secondary images are the result of multiple gratings being illuminated by a single LCD pixel. This causes the corresponding display pixel to appear to be on in virtual viewing slits from which it should appear to be off. When this occurs, a dimmer copy of the 2-D image intended for one virtual viewing slit is visible in other virtual viewing slits. Illumination of multiple gratings by a single LCD pixel can be attributed primarily to (1) diffraction from the LCD pixel aperture as the light propagates from the LCD to the DOE, and (2) divergence of the readout illumination. Electrical crosstalk between LCD pixels can also contribute to the presence of secondary images but this can be effectively minimized by adjusting the brightness and contrast of the LCD.

The degree to which secondary images are visible depends on the separation of the DOE from the LCD. For example, consider the geometry illustrated in Fig. 8. Light going through the LCD pixel is intended to illuminate grating $B$. Assuming that the incident illumination is perfectly collimated and uniform, the intensity at the DOE is given by $^{20}$

$$
\begin{aligned}
I_{\mathrm{DOE}}(x, y)= & \mid \iint \exp \left\{j \frac { \pi } { \lambda z } \left[(x-\eta)^{2}\right.\right. \\
& \left.\left.+(y-\gamma)^{2}\right]\right\}\left.\mathrm{d} \eta \mathrm{d} \gamma\right|^{2},
\end{aligned}
$$

in which the integration is over the clear aperture of the LCD pixel, $\lambda$ is the wavelength of the readout illumination, and $z$ is the optical separation between the aperture plane of the LCD (which is typically on one of the glass surfaces adjacent to the liquid crystal layer) and the DOE. Examination of the $x$-profile of this intensity as shown in Fig. 8 illustrates that a portion of the light that goes through the
LCD pixel is incident on the two gratings $(A$ and $C$ ) that are adjacent to grating $B$. The power incident on each of the gratings is found by integrating the intensity profile given by Eq. (11) over the respective grating apertures. The ratio of the power incident on grating $A$ to the power incident on grating $B$ due to diffraction is $5.3 \%$.

Likewise, divergence in the readout beam illumination can cause light that passes through the LCD pixel of Fig. 8 to illuminate gratings $A$ and $C$ as well as the intended grating $B$. Again, this is a function of the separation between the LCD and the DOE. The divergence of the readout beam in our lab bench setup was approximately $0.5 \mathrm{deg}$. Neglecting the effects of diffraction from the LCD pixel aperture, this causes the amount of power incident on grating $A$ to be $5.6 \%$ as much as is incident on grating $B$. We find that when the readout beam divergence is increased, the brightness of the secondary images also increases, which results in degraded image quality and increased interocular crosstalk.

The use of a redesigned fixture that reduces the separation between the LCD and the DOE from $1 \mathrm{~mm}$ to approximately $250 \mu \mathrm{m}$ results in a noticeable improvement in display quality due to decreased brightness of the secondary images. To completely eliminate the optical crosstalk that causes the secondary images, it is clear that the DOE needs to be integrated directly into the LCD cover glass. This should result in a display that has sharp, crisp 2-D images visible from each virtual viewing slit.

\subsection{Contrast Ratio and Optical Efficiency}

As reported in Ref. 12, the measured contrast ratio of our display is approximately 100. The display's brightness depends on its optical efficiency as well as the illumination power. We define the display's optical efficiency, $\eta$, as that fraction of the illumination power that is directed into the pupil of an observer when the pupil is in the center of any given virtual viewing slit. The display's optical efficiency can therefore be used to relate the desired brightness or luminance of the display to the required illumination power or intensity. For example, the luminance of the display, $L_{v}$, when illuminated by a narrowband source can be written as

$L_{\nu}=K_{\nu} V_{d \lambda} \eta \frac{I_{\text {illum }}}{d \Omega}$,

in which $K_{v}$ is the conversion factor between radiometric and photometric quantities $(673 \mathrm{~lm} / \mathrm{W}), V_{d \lambda}$ is the relative visibility factor for a daylight-adapted human eye at the center wavelength of the narrow-band illumination, ${ }^{21} I_{\text {illum }}$ is the illumination intensity in the plane defined by the back of the display, and $d \Omega$ is the solid angle subtended by the pupil of the eye relative to the display. The display's optical efficiency can be expressed as

$\eta=T_{\mathrm{LCD}} F_{p p} \eta_{g} F_{\text {pupil }}$, 
in which $T_{\mathrm{LCD}}$ is the transmissivity of an LCD pixel, $F_{p p}$ is the geometric fill factor of a partial pixel, $\eta_{g}$ is the +1 order diffraction efficiency of a partial pixel grating, and $F_{\text {pupil }}$ is the fraction of the +1 order that enters the pupil.

The measured transmissivity of the LCD pixels (i.e., including only the clear aperture of the pixels), $T_{\mathrm{LCD}}$, is $10 \%$, while the geometric fill factor is $33 \% .{ }^{12}$ The measured diffraction efficiency of the gratings through a $3.2-\mathrm{mm}$ aperture centered in a virtual viewing slit (and thus representing an observer's pupil) is $2.1 \%$. The calculated fraction of the +1 order that enters a pupil of this size, $F_{\text {pupil }}$, is $22 \%$. The diffraction efficiency of the gratings is thus $9.5 \%$, which compares favorably with the theoretical maximum of $10.1 \%$ for a $50 \%$ duty cycle amplitude grating.

The display's overall measured optical efficiency is $0.07 \%$. Using Eq. (12), this leads to a required illumination intensity of only $4.8 \mu \mathrm{W} / \mathrm{cm}^{2}$ to achieve a luminance of 20 foot-lamberts, which is typical for color monitors. We find that a comfortable display brightness is achieved with a flashlight bulb that we use in a small $(22 \times 28.5 \times 58.4 \mathrm{~cm})$ portable version of the display that is driven with a laptop computer.

\subsection{Viewing Region Intensity Profile}

The physical extent of each virtual viewing slit is determined by diffraction from the apertures of the partial pixels that direct light to that virtual viewing slit. The distribution of light within each virtual viewing slit is affected by whether the optical fields due to individual partial pixels add coherently or incoherently. This in turn depends on the coherence area of the incident illumination at the plane of the display. The estimated size of the coherence area for the illumination systems that we have used is in each case larger than the aperture of a partial pixel $(40 \times 25 \mu \mathrm{m})$ but smaller than or on the order of the size of a display pixel $(220 \times 220 \mu \mathrm{m})$. We can therefore treat diffraction from individual partial pixels as a coherent phenomenon while we will assume for purposes of this analysis that the total intensity in any given virtual viewing slit is an incoherent sum of the intensities from individual partial pixels. This is certainly valid when the coherence area is smaller than the size of a display pixel. A detailed treatment of the case in which the coherence area is the same size or larger is a subject for future research.

Given the above considerations, the intensity in a particular virtual viewing slit, $I_{i}$, can be expressed as (neglecting obliquity factors)

$I_{i}(x, y)=\sum_{j=1}^{N} I_{i j} \operatorname{sinc}^{2}\left[\frac{\pi l_{x}\left(x-x_{i j}^{\prime}\right)}{\lambda D_{\nu}}\right] \operatorname{sinc}^{2}\left[\frac{\pi l_{y}\left(y-y_{i j}^{\prime}\right)}{\lambda D_{\nu}}\right]$,

in which $I_{i j}$ is the peak intensity of the beam from the $j$ 'th partial pixel that diffracts light to the $i$ 'th virtual viewing slit, $\left(x_{i j}^{\prime}, y_{i j}^{\prime}\right)$ is the point at which the center of this beam intersects the plane of the viewing region [given by Eq. (6) for the $i j$ 'th grating having a period of $\Lambda_{i j}+\Delta \Lambda_{i j}$ and an angular orientation of $\left.\theta_{G i j}+\Delta \theta_{i j}\right], l_{x}$ and $l_{y}$ are the width and height, respectively, of each partial pixel aperture, $N$ is the number of partial pixels that diffract light to the $i$ 'th virtual viewing slit (which is the same for every virtual

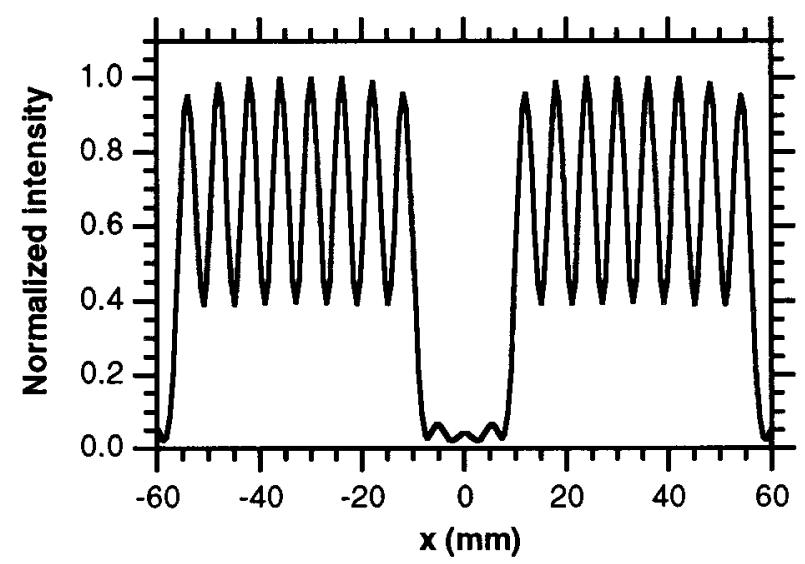

Fig. 9 Expected intensity profile of viewing region along the $x$ axis.

viewing slit), and sinc $\delta \equiv(\sin \delta) / \delta$. For the case of ideal gratings (i.e., no fabrication errors) and uniform readout illumination, Eq. (14) reduces to

$I_{i}(x, y)=I_{0} \operatorname{sinc}^{2}\left[\frac{\pi l_{x}\left(x-x_{i}\right)}{\lambda D_{\nu}}\right] \operatorname{sinc}^{2}\left[\frac{\pi l_{y}\left(y-y_{i}\right)}{\lambda D_{\nu}}\right]$,

in which $\left(x_{i}, y_{i}\right)$ is the center of the $i$ 'th virtual viewing slit and $I_{0}$ is $N$ times the peak intensity due to light from a single partial pixel.

The intensity profile of the entire viewing region is simply the sum of the intensities of the virtual viewing slits. For ideal gratings this is just

$I_{\text {tot }}(x, y)=I_{0} \sum_{i=1}^{M} \operatorname{sinc}^{2}\left[\frac{\pi l_{x}\left(x-x_{i}\right)}{\lambda D_{\nu}}\right] \operatorname{sinc}^{2}\left[\frac{\pi l_{y}\left(y-y_{i}\right)}{\lambda D_{\nu}}\right]$

in which $M$ is the number of virtual viewing slits. A plot of the expected profile along the $x$ axis of the viewing region for our demonstration display is shown in Fig. 9. The center of each virtual viewing slit is located at a corresponding peak in the intensity profile.

A critical design choice is illustrated by examining the positions of the nominal edges of the virtual viewing slits, which are found at the minima between adjacent peaks. As illustrated in Fig. 10, there is inevitably some degree of overlap of light between adjacent virtual viewing slits since each slit intensity has a sinc ${ }^{2}$ profile [Eq. (15)]. If the separation of the virtual viewing slit centers is larger than the null-to-null [i.e., full width full maximum (FWFM)] width of the slit's sinc ${ }^{2}$ profile, discrete slits will be noticeable as the viewer's head horizontally traverses the viewing region. This is similar to looking at a 3-D scene through a picket fence. On the other hand, if the virtual viewing slit centers are separated by only a small fraction of the FWFM sinc ${ }^{2}$ width, 2-D images from nonadjacent slits will be visible simultaneously, which can cause unacceptable crosstalk.

Given the partial pixel width of our display (and hence the FWFM width of the sinc ${ }^{2}$ intensity profile), we arbitrarily chose a virtual viewing slit width $(6 \mathrm{~mm})$ so that the 


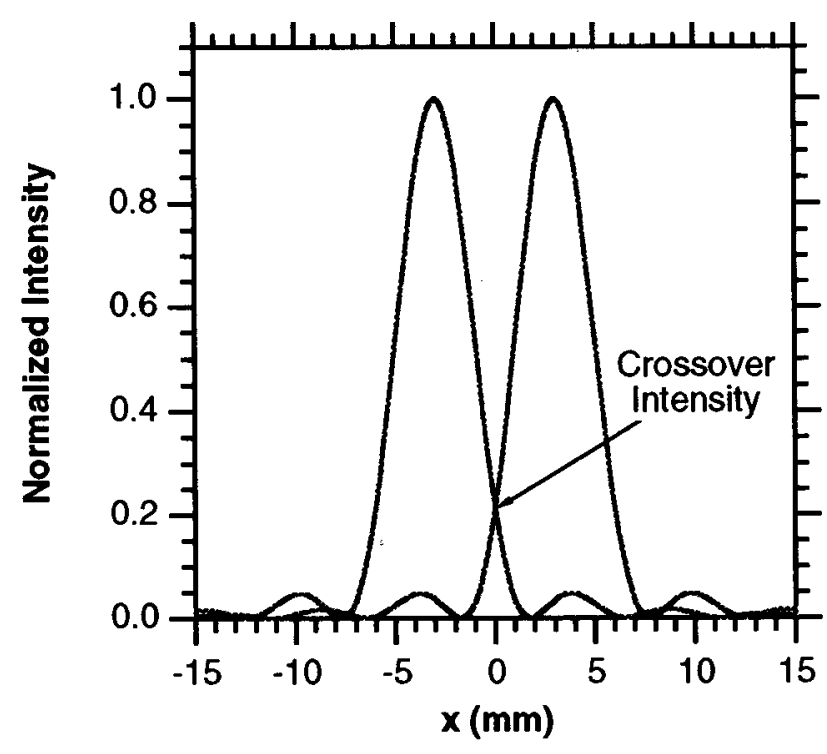

Fig. 10 Individual intensity profiles of adjacent virtual viewing slits.

crossover point between adjacent virtual viewing slits is $21 \%$ of the peak intensity in either slit (as shown in Fig. 10). This results in a minimum intensity between adjacent peaks in Fig. 9 of approximately 39\% of the peak maximum. When viewing the display, this design choice for the crossover intensity does not cause the picket fence effect to be observable, and creates a smooth transition between 2-D images in adjacent virtual viewing slits when the viewer's head is translated horizontally. The visual effects of other specific choices for the crossover intensity are a topic for future research.

The actual intensity profile in the viewing region that is generated by our display was recorded using a CCD camera and is shown in Fig. 11(a). Two lenses were used to demagnify the profile so that it was small enough to be captured in a single CCD image. The associated transverse (x-dimension) profile is shown in Fig. 11(b). Note that the actual profile generated by the display is comparable to the theoretical profile. The average value of the ratio of each minimum to an adjacent peak is $36 \%$, which is close to the designed value for the display. Thus the virtual viewing slits and the viewing region are formed as expected.

\section{Future Research}

We have recently completed a full color version of the display reported here and are currently characterizing it. We are also working on an integrated DOE that includes phase partial pixel gratings and a vertical diffuser to increase the viewing region height. Long-term research efforts will also focus on scaling up the display size, resolution, angular viewing range, and number of virtual viewing slits.

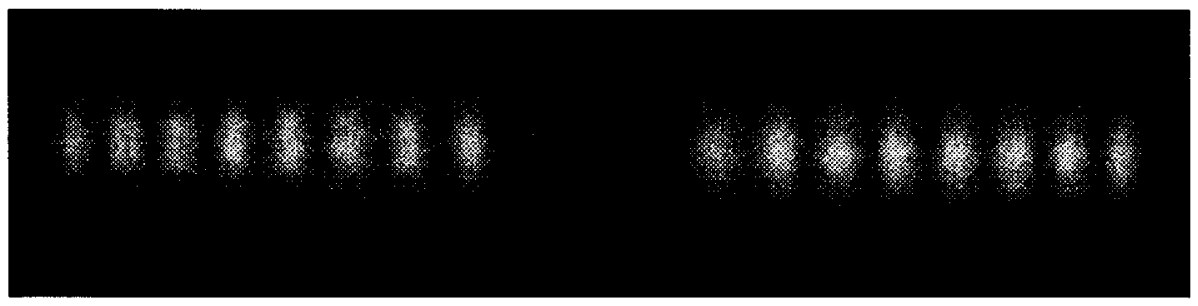

(a)

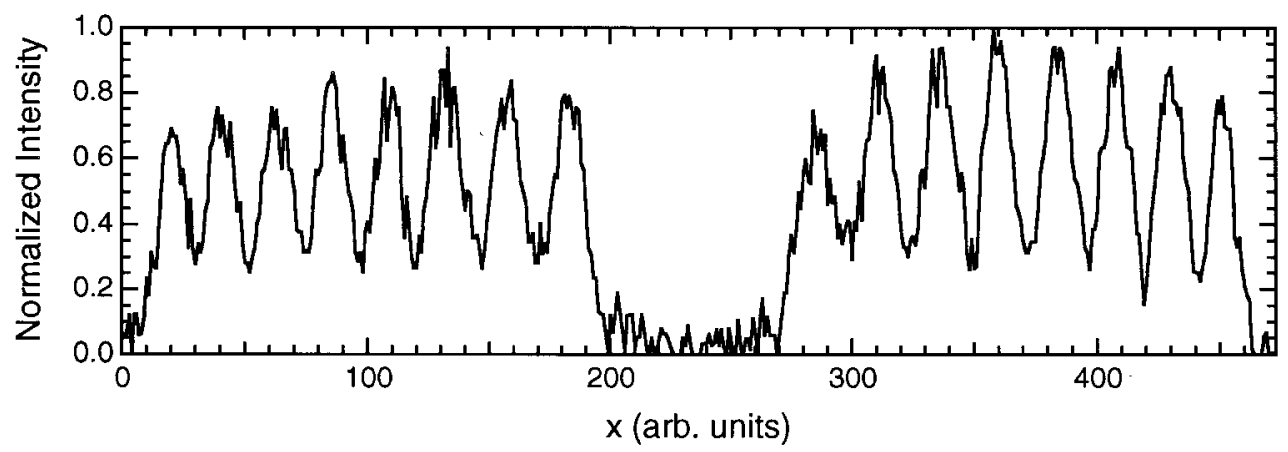

(b)

Fig. 11 (a) Density plot of the measured viewing region intensity; (b) measured $x$ axis intensity profile. 


\section{Acknowledgments}

This project was supported by the Advanced Research Projects Agency and the U.S. Army Missile Command. We thank PhotoSciences, Inc. for their efforts in fulfilling our mask fabrication requirements and Anees Ahmad and Deborah Bailey for their help with the design of the alignment fixture. We also express our appreciation to Ahmed Siddique for his assistance with software development.

\section{References}

1. D. F. McAllister (ed.), Stereo Computer Graphics and Other True 3D Technologies, Princeton Univ. Press, Princeton, NJ (1993).

2. B. Rogers and M. Graham, "Similarities between motion parallax and stereopsis in human depth perception," Vision Res. 22, 261-270 (1982).

3. P. St. Hilaire, S. A. Benton, M. Lucente, M. L. Jepson, J. Kollin, H. Yoshikawa, and J. Underkoffler, "Electronic display system for computational holography,' Proc. SPIE 1212, 174-182 (1990).

4. P. St. Hilaire, S. A. Benton, M. Lucente, H. Yoshikawa, and J. Underkoffler, "Real-time holographic display: improvements using a multichannel acousto-optic modulator and holographic optical elements,'" Proc. SPIE 1461, 256-61 (1991)

5. S. A. Benton, "Experiments in holographic video imaging," Proc. SPIE IS08, 247-267 (1991).

6. P. St. Hilaire, S. A. Benton, and M. Lucente, "Synthetic aperture holography: a novel approach to three-dimensional display,' J. Opt. Soc. Am. A 9(11), 1969-1977 (1992).

7. P. St. Hilaire, "Scalable optical architecture for electronic holography," Opt. Eng. 34(10), 2900-2911 (1995)

8. N. Hashimoto and S. Morokawa, "Real-time electroholographic system using liquid crystal television spatial light modulators,' J. Electr. Imaging 2(2), 93-99 (1993)

9. G. P. Nordin, J. H. Kulick, M. Jones, P. Nasiatka, R. G. Lindquist, and S. T. Kowel, "Demonstration of a novel 3-D autostereoscopic display,', Opt. Lett. 19(12), 901-903 (1994).
10. J. H. Kulick, G. P. Nordin, A. Parker, S. T. Kowel, R. G. Lindquist, M. Jones, and P. Nasiatka, "Partial pixels: a three-dimensional diffractive display architecture,', J. Opt. Soc. Am A 12(1), 73-83 (1995)

11. G. P. Nordin, J. H. Kulick, R. G. Lindquist, P. J. Nasiatka, M. W. Jones, M. Friends, and S. T. Kowel, "Liquid crystal-on-silicon implementation of the partial pixel three-dimensional display architecture,' Appl. Opt. 34(19), 3756-3763 (1995).

12. M. W. Jones, G. P. Nordin, J. H. Kulick, R. G. Lindquist, and S. T. Kowel, "Real-time three-dimensional display based on the partial pixel architecture,' Opt. Lett. 20(12), 1418-1420 (1995).

13. T. Toda, S. Takahashi, and F. Iwata, "Three-dimensional video system using grating image," Proc. SPIE 2406, 191-198 (1995).

14. G. P. Nordin, M. W. Jones, J. H. Kulick, R. G. Lindquist, and S. T Kowel, "Implementation of a full-color partial pixel display,", OSA Annual Meeting and Exhibit 1995, p. 111, Optical Society of America, Washington, DC (1995).

15. J. H. Kulick, M. W. Jones, G. P. Nordin, R. G. Lindquist, S. T Kowel, and A. Thomsen, "Demonstration of a real-time implementation of the ICVision holographic stereogram display," Proc. SPIE 2577, 8-19 (1995)

16. M. Thompson, "The creation of new information paradigms through flat panel displays,', OSA Annual Meeting and Exhibit 1995, p. 73 , Optical Society of America, Washington, DC (1995).

17. R. G. Lindquist, J. H. Kulick, G. P. Nordin, J. M. Jarem, S. T. Kowel, M. Friends, and T. M. Leslie, "High-resolution liquid crystal phase grating formed by fringing fields from interdigitated electrodes," Opt. Lett. 19(9), 670-672 (1994).

18. R. Horstman, personal communication (1995).

19. Y.-Y. Yeh and L. D. Silverstein, "Limits of fusion and depth judgement in stereoscopic displays," Hum. Factors 32, 45-60 (1990).

20. J. W. Goodman, Introduction to Fourier Optics, McGraw-Hill, San Francisco (1968)

21. M. Born and E. Wolf, Principles of Optics, Pergamon Press, New York (1980).

Biographies and photographs of the authors not available. 